\title{
Reliability study of proportional odds family of discrete distributions
}

\author{
Pradip Kundu and Asok K. Nanda* \\ Department of Mathematics and Statistics, IISER Kolkata \\ Mohanpur 741246, India
}

\begin{abstract}
The proportional odds model gives a method of generating new family of distributions by adding a parameter, called tilt parameter, to expand an existing family of distributions. The new family of distributions so obtained is known as Marshall-Olkin family of distributions or Marshall-Olkin extended distributions. In this paper, we consider Marshall-Olkin family of distributions in discrete case with fixed tilt parameter. We study different ageing properties, as well as different stochastic orderings of this family of distributions. All the results of this paper are supported by several examples.
\end{abstract}

Keywords: Marshall-Olkin extended distribution, Stochastic ageing, Stochastic orders.

\section{Introduction}

Since the work of Bennett [1] and Pettitt [25] on the proportional odds (PO) model in the survival analysis context, it has been used by many researchers. The assumption of constant hazard ratio is unreasonable in many practical cases as discussed by Bennett [1, Kirmani and Gupta [17] and Rossini and Tsiatis [26]. The PO model has been used by Bennett [1] to demonstrate the effectiveness of a cure, when the mortality rate of a group having some disease approaches that of a (disease-free) control group as time progresses. After Bennet's [1] work, the PO model has found many practical applications, see, for instance, Collett [4], Dinse and Lagakos [8], Pettitt [25] and Rossini and Tsiatis [26]. Let $X$ and $Y$ be two random variables with distribution functions $F(\cdot), G(\cdot)$, survival functions $\bar{F}(\cdot), \bar{G}(\cdot)$, probability density functions $f(\cdot), g(\cdot)$ and hazard rate functions $r_{X}(\cdot)=f(\cdot) / \bar{F}(\cdot), r_{Y}(\cdot)=g(\cdot) / \bar{G}(\cdot)$. Let the odds functions of $X$ and $Y$ be denoted respectively by $\theta_{X}(t)=\bar{F}(t) / F(t)$ and $\theta_{Y}(t)=\bar{G}(t) / G(t)$. The random variables $X$ and $Y$ are said to satisfy PO model with proportionality constant $\alpha$ if

${ }^{*}$ Corresponding author, e-mail: asok.k.nanda@gmail.com; asok@iiserkol.ac.in 
$\theta_{Y}(t)=\alpha \theta_{X}(t)$. For more discussion on PO models one may refer to Kirmani and Gupta [17]. It is observed that, in terms of survival functions, the PO model can be represented as

$$
\bar{G}(t)=\frac{\alpha \bar{F}(t)}{1-\bar{\alpha} \bar{F}(t)},
$$

where $\bar{\alpha}=1-\alpha$. From the above representation we have

$$
\frac{r_{Y}(t)}{r_{X}(t)}=\frac{1}{1-\bar{\alpha} \bar{F}(t)}=\frac{G(t)}{F(t)},
$$

so that the hazard ratio is increasing (resp. decreasing) for $\alpha>1$ (resp. $\alpha<1$ ) and it convergence to 1 as $t$ tends to $\infty$. This property of hazard functions makes the PO model reasonable in many practical applications. This is in contrast to the proportional hazards model where the ratio of the hazard rates remains constant with time.

The model (1.1), with $0<\alpha<\infty$; gives a method of introducing new parameter $\alpha$ to a family of distributions for obtaining more flexible new family of distributions as discussed by Marshall and Olkin [19]. The family of distributions so obtained is known as Marshall-Olkin family of distributions or Marshall-Olkin extended distributions (for details see [19, 20]). For more discussion and applications of Marshall-Olkin family of distributions one can see [3, 5, 6, 13. The parameter $\alpha$ is called 'tilt parameter'. This is because the hazard rate of the new family is shifted below or above the hazard rate of the underlying (baseline) distribution for $\alpha \geq 1$ and $0<\alpha \leq 1$ respectively. Thus, Marshall-Olkin family of distributions has implications both in terms of PO model as well as in generating a new family of flexible distributions, and hence it is worth to investigate this family of distributions.

Kirmani and Gupta [17] studied some ageing properties of the PO model with fixed tilt parameter $\alpha$. Nanda and Das [22] studied different ageing classes of Marshall-Olkin family of distributions taking the tilt parameter as random variable. Ghitany and Kotz [14] studied the reliability properties by taking $\bar{F}$ as the reliability function of the linear failure-rate distribution. Gupta et al. [16] compared the Marshall-Olkin extended distribution and the original distribution with respect to some stochastic orderings. Nanda and Das [23] compared this family of distributions with respect to different stochastic orderings by taking the tilt parameter random. All the studies mentioned above consider the original (baseline) distribution to be continuous. However, not much work is available in the literature for discrete case. In this paper, we study different ageing properties, as well as different stochastic orderings of this family of distributions with discrete baseline distribution and with fixed tilt parameter.

\section{Preliminaries}

Here we discuss the survival function, the hazard (failure) rate function, and the mean residual life of a discrete random variable $X$ with support $\mathbb{N}=\{1,2, \ldots\}$. Let the probability mass 
function (pmf) of $X$ be given by $f(k)=P\{X=k\}$, and the distribution function be $F(\cdot)$ so that the reliability (survival) function $\bar{F}(\cdot)$ of $\mathrm{X}$ becomes

$$
\bar{F}(k)=P\{X>k\}=\sum_{j=k+1}^{\infty} f(j), k=1,2, \ldots,
$$

with $\bar{F}(0)=1$. The failure rate function $r(\cdot)$ (Shaked et al. [28]) is given by

$$
r(k)=P\{X=k \mid X \geq k\}=\frac{P\{X=k\}}{P\{X \geq k\}}=\frac{f(k)}{\bar{F}(k-1)},
$$

and the reversed hazard rate function is given by $\tilde{r}(k)=f(k) / F(k)$. Below we give the definitions of different discrete ageing classes.

Definition 2.1 A discrete random variable $X$ is said to be

(i) ILR (DLR) i.e. increasing (decreasing) in likelihood ratio if $f(k)$ is log-concave (logconvex), i.e. if $f(k+2) f(k) \leq(\geq) f^{2}(k+1), k \in \mathbb{N}$ (Dewan and Sudheesh [9]);

(ii) IFR (DFR) i.e. increasing (decreasing) failure rate if $r(k)$ is increasing (decreasing) in $k \in \mathbb{N}$. This is equivalent to the fact that $\bar{F}(k+1) / \bar{F}(k)$ is decreasing (increasing) in $k \in \mathbb{N}$ (Salvia and Bollinger [27], Shaked et al. [28], Gupta et al. [15]);

(iii) IFRA (DFRA) i.e. increasing (decreasing) in failure rate average if $[\bar{F}(k)]^{1 / k}$ is decreasing (increasing) in $k$, i.e., $[\bar{F}(k)]^{1 / k} \geq(\leq)[\bar{F}(k+1)]^{1 /(k+1)}, k \in \mathbb{N}$ (Esary et al. [10], Shaked et al. [28]);

(iv) $N B U$ (NWU) i.e. new better (worse) than used if $\bar{F}(j+k) \leq(\geq) \bar{F}(j) \bar{F}(k), j, k \in \mathbb{N}$ (Esary et al. [10], Shaked et al. [28]);

(v) DRHR (decreasing reversed hazard rate) if $\tilde{r}(k)$ is decreasing in $k$, i.e. if $F(k)$ is $\log$ concave, i.e. if $[F(k+1)]^{2} \geq F(k) F(k+2), k \in \mathbb{N}$ (Nanda and Sengupta 24], Li and Xu [18]).

(vi) NBAFR (new better than used in failure rate average) if $[\bar{F}(k)]^{1 / k} \leq \bar{F}(1), k \in \mathbb{N}$ (Fagiuoli and Pellerey [11]).

\subsection{Proportional odds family of discrete distributions}

Let $X$ be a discrete random variable with support $\mathbb{N}=\{1,2, \ldots\}$ having pmf $f(\cdot)$, distribution function $F(\cdot)$, survival function $\bar{F}(\cdot)$, hazard rate function $r_{X}(\cdot)$, and reversed hazard rate function $\tilde{r}_{X}(\cdot)$. Starting with the survival function $\bar{F}$, the survival function of the proportional odds family (also known as Marshall-Olkin family) of discrete distribution is given by

$$
\bar{G}(k ; \alpha)=\frac{\alpha \bar{F}(k)}{1-\bar{\alpha} \bar{F}(k)}, k=1,2, \ldots, 0<\alpha<\infty, \bar{\alpha}=1-\alpha,
$$


with $\bar{G}(0 ; \alpha)=1$. Let the corresponding random variable be denoted by $Y$. Now the distribution function of $Y$ is given by

$$
G(k ; \alpha)=1-\bar{G}(k ; \alpha)=\frac{F(k)}{1-\bar{\alpha} \bar{F}(k)}
$$

whereas the pmf is given by

$$
g(k ; \alpha)=\bar{G}(k-1 ; \alpha)-\bar{G}(k ; \alpha)=\frac{\alpha f(k)}{[1-\bar{\alpha} \bar{F}(k-1)][1-\bar{\alpha} \bar{F}(k)]} .
$$

The corresponding hazard rate and the reversed hazard rate functions are given by

$$
\begin{gathered}
r_{Y}(k ; \alpha)=\frac{r_{X}(k)}{1-\bar{\alpha} \bar{F}(k)}, \\
\tilde{r}_{Y}(k ; \alpha)=\frac{\alpha \tilde{r}_{X}(k)}{1-\bar{\alpha} \bar{F}(k-1)} .
\end{gathered}
$$

It is to be mentioned here that different properties of (2.1) have been studied by Déniz and Sarabia [7] by taking $F$ as the cdf of Poisson random variable.

\section{$3 \quad$ Stochastic Ageing properties}

In this section we study how different ageing properties of $X$ are transmitted to the random variable $Y$.

With the following two counterexamples, one with $\alpha>1$ and the other with $\alpha<1$, we show that if $X$ is ILR, then $Y$ is neither ILR nor DLR.

Counterexample 3.1 Consider the random variable $X$ with the mass function given by

$$
f(k)= \begin{cases}0, & \text { if } k=1 \\ 0.1, & \text { if } k=2 \\ 0.25, & \text { if } k=3 \\ 0.35, & \text { if } k=4 \\ 0.3, & \text { if } k=5\end{cases}
$$

Clearly $X$ is ILR. For $\alpha=5$, we have the mass function of $Y$ as

$$
g(k ; 5)= \begin{cases}0, & \text { if } k=1 ; \\ \frac{1}{46}, & \text { if } k=2 ; \\ \frac{125}{1656}, & \text { if } k=3 ; \\ \frac{175}{792}, & \text { if } k=4 ; \\ \frac{15}{22}, & \text { if } k=5 .\end{cases}
$$

It is observed that $Y$ is neither ILR nor DLR. 
Counterexample 3.2 Consider the random variable $X$ with mass function given by

$$
f(k)= \begin{cases}0, & \text { if } k=1 \\ 0.3, & \text { if } k=2 \\ 0.34, & \text { if } k=3 \\ 0.26, & \text { if } k=4 \\ 0.1, & \text { if } k=5\end{cases}
$$

Here $X$ is ILR. For $\alpha=0.2$, we have the mass function of $Y$ as

$$
g(k ; 0.2)= \begin{cases}0, & \text { if } k=1 \\ \frac{15}{22}, & \text { if } k=2 ; \\ \frac{425}{1958}, & \text { if } k=3 \\ \frac{325}{409}, & \text { if } k=4 ; \\ \frac{1}{46}, & \text { if } k=5\end{cases}
$$

It is observed that $Y$ is neither ILR nor DLR.

With the following two counterexamples, one for $\alpha>1$ and the other for $\alpha<1$, we show that if $X$ is DLR, then $Y$ is neither DLR nor ILR.

Counterexample 3.3 Consider the random variable $X$ with mass function given by

$$
f(k)= \begin{cases}0.36, & \text { if } k=1 \\ 0.26, & \text { if } k=2 \\ 0.21, & \text { if } k=3 \\ 0.17, & \text { if } k=4\end{cases}
$$

Here $X$ is DLR. For $\alpha=2$, we have the mass function of $Y$ as

$$
g(k ; 2)= \begin{cases}\frac{9}{41}, & \text { if } k=1 ; \\ \frac{650}{2529}, & \text { if } k=2 ; \\ \frac{700}{2691}, & \text { if } k=3 ; \\ \frac{34}{117}, & \text { if } k=4 .\end{cases}
$$

It is observed that $Y$ is neither DLR nor ILR.

Counterexample 3.4 Consider the random variable $X$ with mass function given by

$$
f(k)= \begin{cases}0.26, & \text { if } k=1 \\ 0.18, & \text { if } k=2 \\ 0.24, & \text { if } k=3 \\ 0.32, & \text { if } k=4\end{cases}
$$


Here $X$ is DLR. For $\alpha=0.4$, we have the mass function of $Y$ as

$$
g(k ; 0.4)= \begin{cases}\frac{65}{139}, & \text { if } k=1 ; \\ \frac{2250}{11537}, & \text { if } k=2 ; \\ \frac{1500}{8383}, & \text { if } k=3 ; \\ \frac{16}{101}, & \text { if } k=4 .\end{cases}
$$

It is observed that $Y$ is neither DLR nor ILR.

The following theorem gives the condition under which IFR/DFR property of $X$ is transmitted to the random variable $Y$. The proof follows from the fact that $1 /(1-\bar{\alpha} \bar{F}(k))$ is increasing (resp. decreasing) in $k$ for $\alpha \geq($ resp. $\leq) 1$.

Theorem 3.1 If $X$ is IFR (resp. DFR) and $\alpha \geq($ resp. $\leq)$, then $Y$ is IFR (resp. DFR).

The following counterexample shows that if $\alpha<1$, then the IFR property of $X$ may not be transmitted to the random variable $Y$.

Counterexample 3.5 Consider the random variable $X$ following discrete IFR distribution (cf. Salvia and Bollinger [27]) with

$$
f(k)=(k-c) c^{k-1} / k !, k \in \mathbb{N}, 0<c \leq 1 .
$$

Here $\bar{F}(k)=c^{k} / k$ ! and $r_{X}(k)=1-c / k$ so that $X$ is IFR. Now

$$
r_{Y}(k ; \alpha)=\frac{1-c / k}{1-\bar{\alpha} c^{k} / k !} .
$$

It is observed that, for $c=0.8$ and $\alpha=0.2$, we have $r_{Y}(2 ; 0.2)=0.8064516, r_{Y}(3 ; 0.2)=$ 0.7870635 , and $r_{Y}(4 ; 0.2)=0.8110739$. This shows that $Y$ is neither IFR nor DFR.

The following counterexample shows that the DFR property of $X$ may not be transmitted to the random variable $Y$ when $\alpha>1$.

Counterexample 3.6 Let $X$ follow the Type I discrete Weibull distribution (cf. Nakagawa and Osaki [21]) with pmf given by

$$
f(k)=q^{(k-1)^{\beta}}-q^{k^{\beta}}, k \in \mathbb{N}, q \in(0,1), \beta>0 .
$$

Then the corresponding survival function is given by $\bar{F}(k)=q^{k^{\beta}}$, and the hazard rate function is given by $r_{X}(k)=1-q^{k^{\beta}-(k-1)^{\beta}}$. Here $X$ is DFR for $0<\beta<1$. Note that

$$
r_{Y}(k ; \alpha)=\frac{1-q^{k^{\beta}-(k-1)^{\beta}}}{1-\bar{\alpha} q^{k^{\beta}}} .
$$

It is observed that, for $\beta=0.8, \alpha=5$ and $q=0.5$, we have $r_{Y}(7 ; 5)=0.2759209, r_{Y}(10 ; 5)=$ 0.2834942 , and $r_{Y}(13 ; 5)=0.2793229$. This shows that $Y$ is neither DFR nor IFR. 
Below we see that, for $\alpha \geq($ resp. $\leq$ ) 1, the NBU (resp. NWU) property of $X$ is transmitted to the random variable $Y$.

Theorem 3.2 If $X$ is $N B U$ (resp. NWU) and $\alpha \geq($ resp. $\leq) 1$, then $Y$ is $N B U$ (resp. $N W U$ ).

Proof: Let $X$ be NBU (resp. NWU). Then $Y$ will be NBU (resp. NWU) if and only if

$$
\frac{1-\bar{\alpha} \bar{F}(j+k)}{\bar{F}(j+k)} \geq(\text { resp. } \leq) \frac{(1-\bar{\alpha} \bar{F}(j))(1-\bar{\alpha} \bar{F}(k))}{\alpha \bar{F}(j) \bar{F}(k)} .
$$

This is equivalent to the fact that

$$
\frac{1}{\bar{F}(j+k)} \geq(\text { resp. } \leq) \frac{1-\bar{\alpha}(\bar{F}(j)+\bar{F}(k))+\bar{\alpha} \bar{F}(k) \bar{F}(j)}{\alpha \bar{F}(j) \bar{F}(k)},
$$

which holds if

$$
\frac{1}{\bar{F}(j+k)} \geq(\text { resp. } \leq) \frac{1}{\bar{F}(j) \bar{F}(k)} .
$$

The last inequality follows from the fact that, for $\alpha \geq($ resp. $\leq) 1$,

$$
1-\bar{\alpha}(\bar{F}(j)+\bar{F}(k))+\bar{\alpha} \bar{F}(k) \bar{F}(j) \leq(\text { resp. } \geq) \alpha .
$$

Hence the theorem follows.

The following counterexamples show that, for $\alpha<$ (resp. $>$ ) 1, the NBU (resp. NWU) property of $X$ may not be transmitted to the random variable $Y$.

Counterexample 3.7 Consider $X$ following the discrete $S$-distribution (cf. Bracuemond and Gaudoin [2]) with pmf given by

$$
f(k)=p\left(1-a^{k}\right) \prod_{i=1}^{k-1}\left(1-p+p a^{i}\right), k \in \mathbb{N}, 0<p \leq 1,0<a<1 .
$$

This gives the survival function as $\bar{F}(k)=\prod_{i=1}^{k}\left(1-p+p a^{i}\right)$, and hazard rate function as $r_{X}(k)=p\left(1-a^{k}\right)$. Here $X$ is NBU. Now

$$
\bar{G}(k ; \alpha)=\frac{\alpha \prod_{i=1}^{k}\left(1-p+p a^{i}\right)}{1-\bar{\alpha} \prod_{i=1}^{k}\left(1-p+p a^{i}\right)} .
$$

For $j=2, k=3, p=0.3, a=0.6, \alpha=0.2$, we have $\bar{G}(j+k ; \alpha)=0.075737$ and $\bar{G}(j ; \alpha) \bar{G}(k ; \alpha)=0.063494$. This shows that $Y$ is not $N B U$.

Counterexample 3.8 Let $X$ follow the distribution as given in Counterexample [3.6. Then clearly $X$ is $N W U$ for $\beta \in(0,1)$. Now, for $j=2, k=3, \alpha=5, q=0.5$, we have $\bar{G}(j+k ; \alpha)=$ 0.3062174 and $\bar{G}(j ; \alpha) \bar{G}(k ; \alpha)=0.3657684$. This shows that $Y$ is not $N W U$. 
Kirmani and Gupta [17] have observed that if $X$ is IFRA (DFRA), then $Y$ is IFRA (DFRA) for $\alpha>(<) 1$. Below we show that if $X$ is IFRA, then $Y$ may not be IFRA or DFRA when $\alpha<1$.

Counterexample 3.9 Let $X$ follow the distribution as given in Counterexample 3.7. Here $X$ is IFRA. Now, for $\alpha=0.2, p=0.5$, and $a=0.6$, we have

$$
[\bar{G}(k ; 0.2)]^{1 / k}= \begin{cases}0.44444, & \text { for } k=1 \\ 0.438901, & \text { for } k=2 ; \\ 0.457806, & \text { for } k=4 .\end{cases}
$$

This shows that $Y$ is neither IFRA nor DFRA.

Below we show that if $X$ is DFRA, then, for $\alpha>1, Y$ may not be DFRA or IFRA.

Counterexample 3.10 Let $X$ follow the discrete Pareto distribution with survival function

$$
\bar{F}(k)=\left(\frac{d}{k+d}\right)^{c}, k \in \mathbb{N}, c, d>0,
$$

which is DFR and hence DFRA. Now

$$
\bar{G}(k ; \alpha)=\frac{\alpha\left(\frac{d}{k+d}\right)^{c}}{1-\bar{\alpha}\left(\frac{d}{k+d}\right)^{c}} .
$$

For $\alpha=6, d=2, c=3$, we have

$$
[\bar{G}(k ; 6)]^{1 / k}= \begin{cases}0.7164179, & \text { for } k=1 ; \\ 0.658037, & \text { for } k=4 ; \\ 0.68081, & \text { for } k=8,\end{cases}
$$

which is neither increasing nor decreasing in $k$, i.e. $Y$ is neither DFRA nor IFRA.

Following theorem shows that, for $\alpha \leq 1$, the DRHR property of $X$ is transmitted to the random variable $Y$. The proof follows from the fact that $1 /(1-\bar{\alpha} \bar{F}(k-1))$ is decreasing in $k$, for $\alpha \leq 1$.

Theorem 3.3 If $X$ is DRHR, then $Y$ is DRHR for $\alpha \leq 1$.

The following counterexample shows that, for $\alpha>1$, DRHR property of $X$ may not be transmitted to the random variable $Y$.

Counterexample 3.11 Consider the random variable $X$ having distribution function given by

$$
F(k)= \begin{cases}0, & \text { if } 1 \leq k<2 ; \\ \frac{4}{25}, & \text { if } 2 \leq k<3 ; \\ \frac{2}{5}, & \text { if } 3 \leq k<4 ; \\ \frac{2}{3}, & \text { if } 4 \leq k<5 ; \\ 1, & \text { if } k \geq 5 .\end{cases}
$$


Clearly $X$ is DRHR. For $\alpha=4$, the distribution function of $Y$ is given by

$$
G(k ; 4)= \begin{cases}0, & \text { if } 1 \leq k<2 ; \\ \frac{1}{22}, & \text { if } 2 \leq k<3 \\ \frac{1}{7}, & \text { if } 3 \leq k<4 ; \\ \frac{1}{3}, & \text { if } 4 \leq k<5 ; \\ 1, & \text { if } k \geq 5,\end{cases}
$$

which is not DRHR.

The following counterexample shows that, for $\alpha<1$, NBAFR property of $X$ may not be transmitted to the random variable $Y$.

Counterexample 3.12 Consider the random variable $X$ with reliability function given by

$$
\bar{F}(k)= \begin{cases}\frac{4}{5}, & \text { if } 1 \leq k<2 \\ \frac{8}{13}, & \text { if } 2 \leq k<3 \\ \frac{1}{2}, & \text { if } 3 \leq k<4 \\ 0, & \text { if } k \geq 4\end{cases}
$$

Here $X$ is NBAFR. For $\alpha=0.4$, we have the reliability function of $Y$ as

$$
\bar{G}(k ; 0.4)= \begin{cases}\frac{8}{13}, & \text { if } 1 \leq k<2 ; \\ \frac{16}{41}, & \text { if } 2 \leq k<3 ; \\ \frac{2}{7}, & \text { if } 3 \leq k<4 ; \\ 0, & \text { if } k \geq 4 .\end{cases}
$$

It is clear that $Y$ is not NBAFR.

We summarize the above findings in Table 1.

\section{Stochastic Orderings}

Let $X_{1}$ and $X_{2}$ be two discrete random variables with support $\mathbb{N}=\{1,2, \ldots\}$ having respective pmf $f_{1}(\cdot), f_{2}(\cdot)$, distribution function $F_{1}(\cdot), F_{2}(\cdot)$, and survival function $\bar{F}_{1}(\cdot), \bar{F}_{2}(\cdot)$. Let the survival function of the Marshall-Olkin family of discrete distributions be given by

$$
\bar{G}_{i}(k ; \alpha)=\frac{\alpha \bar{F}_{i}(k)}{1-\bar{\alpha} \bar{F}_{i}(k)}, 0<\alpha<\infty, \bar{\alpha}=1-\alpha,
$$

and let the corresponding random variable be $Y_{i}, i=1,2$. The following theorem shows that the usual stochastic order between $X_{1}$ and $X_{2}$ and that of $Y_{1}$ and $Y_{2}$ are equivalent.

Theorem 4.1 $Y_{1} \leq_{s t} Y_{2}$ if and only if $X_{1} \leq_{s t} X_{2}$. 
Table 1: Preservation of ageing classes

\begin{tabular}{|l|l|l|}
\hline Ageing properties & \multicolumn{1}{|c|}{$\alpha<1$} & \multicolumn{1}{|c|}{$\alpha>1$} \\
\hline ILR & Not Preserved & Not Preserved \\
DLR & Not Preserved & Not Preserved \\
IFR & Not Preserved & Preserved \\
DFR & Preserved & Not Preserved \\
NBU & Not Preserved & Preserved \\
NWU & Preserved & Not Preserved \\
IFRA & Not Preserved & Preserved \\
DFRA & Preserved & Not Preserved \\
DRHR & Preserved & Not Preserved \\
NBAFR & Not Preserved & \\
\hline
\end{tabular}

Proof: Note that $Y_{1} \leq_{s t} Y_{2}$ if, and only if

$$
\frac{\alpha \bar{F}_{1}(k)}{1-\bar{\alpha} \bar{F}_{1}(k)} \leq \frac{\alpha \bar{F}_{2}(k)}{1-\bar{\alpha} \bar{F}_{2}(k)},
$$

which is equivalent to the fact that $\bar{F}_{1}(k) \leq \bar{F}_{2}(k)$. Hence the theorem follows.

The following theorem gives condition on $\alpha$, under which hazard rate order between $X_{1}$ and $X_{2}$ is transmitted to that between $Y_{1}$ and $Y_{1}$.

Theorem 4.2 If $X_{1} \leq_{h r} X_{2}$, then $Y_{1} \leq_{h r} Y_{2}$, provided $\alpha \geq 1$.

Proof: Since hazard rate order is stronger than usual stochastic order, we have, for $\alpha \geq 1$,

$$
\frac{1}{1-\bar{\alpha} \bar{F}_{1}(k)} \geq \frac{1}{1-\bar{\alpha} \bar{F}_{2}(k)} .
$$

Now, using the hypothesis we have, from (2.4),

$$
r_{Y_{1}}(k ; \alpha)=\frac{r_{X_{1}}(k)}{1-\bar{\alpha} \bar{F}_{1}(k)} \geq \frac{r_{X_{2}}(k)}{1-\bar{\alpha} \bar{F}_{2}(k)}=r_{Y_{2}}(k ; \alpha) .
$$

Hence the theorem follows.

The following counterexample shows that the above theorem does not hold if $\alpha<1$.

Counterexample 4.1 Consider the random variables $X_{1}$ and $X_{2}$ with respective reliability function

$$
\bar{F}_{1}(k)= \begin{cases}1, & \text { if } 1 \leq k<2 ; \\ \frac{1}{2}, & \text { if } 2 \leq k<3 \\ \frac{2}{5}, & \text { if } 3 \leq k<4 \\ 0, & \text { if } k \geq 4,\end{cases}
$$


and

$$
\bar{F}_{2}(k)= \begin{cases}1, & \text { if } 1 \leq k<2 \\ \frac{5}{8}, & \text { if } 2 \leq k<3 \\ \frac{11}{20}, & \text { if } 3 \leq k<4 \\ 0, & \text { if } k \geq 4\end{cases}
$$

This shows that $X_{1} \leq_{h r} X_{2}$. For $\alpha=0.2$, we have the respective reliability function of $Y_{1}$ and $Y_{2}$ as

$$
\bar{G}_{1}(k ; \alpha)= \begin{cases}1, & \text { if } 1 \leq k<2 ; \\ \frac{1}{6}, & \text { if } 2 \leq k<3 \\ \frac{2}{17}, & \text { if } 3 \leq k<4 \\ 0, & \text { if } k \geq 4,\end{cases}
$$

and

$$
\bar{G}_{2}(k ; \alpha)= \begin{cases}1, & \text { if } 1 \leq k<2 \\ \frac{1}{4}, & \text { if } 2 \leq k<3 \\ \frac{11}{56}, & \text { if } 3 \leq k<4 \\ 0, & \text { if } k \geq 4\end{cases}
$$

This shows that $Y_{1} \not \varliminf_{h r} Y_{2}$.

The following theorem gives the condition on $\alpha$ such that reversed hazard rate order between $X_{1}$ and $X_{2}$ is transmitted to that between $Y_{1}$ and $Y_{2}$.

Theorem 4.3 If $X_{1} \leq_{r h r} X_{2}$, then $Y_{1} \leq_{r h r} Y_{2}$, for $0<\alpha \leq 1$.

Proof: Since reversed hazard rate order is stronger than usual stochastic order, we have, for $\alpha \leq 1$,

$$
\frac{1}{1-\bar{\alpha} \bar{F}_{1}(k-1)} \leq \frac{1}{1-\bar{\alpha} \bar{F}_{2}(k-1)}
$$

Now, using the hypothesis we have, from (2.5),

$$
\tilde{r}_{Y_{1}}(k ; \alpha)=\frac{\alpha \tilde{r}_{X_{1}}(k)}{1-\bar{\alpha} \bar{F}_{1}(k-1)} \leq \frac{\alpha \tilde{r}_{X_{2}}(k)}{1-\bar{\alpha} \bar{F}_{2}(k-1)}=\tilde{r}_{Y_{2}}(k ; \alpha)
$$

Hence the theorem follows.

That the above theorem does not hold in case of $\alpha>1$ is shown in the following counterexample.

Counterexample 4.2 Consider the random variables $X_{1}$ and $X_{2}$ with respective distribution function

$$
F_{1}(k)= \begin{cases}0, & \text { if } 1 \leq k<2 ; \\ \frac{5}{24}, & \text { if } 2 \leq k<3 \\ \frac{1}{2}, & \text { if } 3 \leq k<4 \\ \frac{3}{4}, & \text { if } 4 \leq k<5 \\ 1, & \text { if } k \geq 5\end{cases}
$$


and

$$
F_{2}(k)= \begin{cases}0, & \text { if } 1 \leq k<2 \\ \frac{1}{6}, & \text { if } 2 \leq k<3 \\ \frac{5}{12}, & \text { if } 3 \leq k<4 \\ \frac{2}{3}, & \text { if } 4 \leq k<5 \\ 1, & \text { if } k \geq 5\end{cases}
$$

Clearly $X_{1} \leq_{r h r} X_{2}$. For $\alpha=4$, we have the distribution functions of $Y_{1}$ and $Y_{2}$ respectively as

$$
G_{1}(k ; 4)= \begin{cases}0, & \text { if } 1 \leq k<2 \\ \frac{5}{81}, & \text { if } 2 \leq k<3 \\ \frac{1}{5}, & \text { if } 3 \leq k<4 \\ \frac{3}{7}, & \text { if } 4 \leq k<5 \\ 1, & \text { if } k \geq 5\end{cases}
$$

and

$$
G_{2}(k ; 4)= \begin{cases}0, & \text { if } 1 \leq k<2 ; \\ \frac{1}{21}, & \text { if } 2 \leq k<3 \\ \frac{5}{33}, & \text { if } 3 \leq k<4 ; \\ \frac{1}{3}, & \text { if } 4 \leq k<5 ; \\ 1, & \text { if } k \geq 5 .\end{cases}
$$

This shows that $Y_{1} \not \mathbf{x}_{r h r} Y_{2}$.

Following two counterexamples show that the likelihood ratio order between $X_{1}$ and $X_{2}$ is not necessarily transmitted to that between $Y_{1}$ and $Y_{2}$.

Counterexample 4.3 Let $X_{1}$ and $X_{2}$ have the respective probability mass function

$$
f_{1}(k)= \begin{cases}0, & \text { if } k=1 \\ 0.3, & \text { if } k=2 \\ 0.4, & \text { if } k=3 \\ 0.2, & \text { if } k=4 \\ 0.1, & \text { if } k=5\end{cases}
$$

and

$$
f_{2}(k)= \begin{cases}0, & \text { if } k=1 \\ 0.2, & \text { if } k=2 \\ 0.3, & \text { if } k=3 \\ 0.2, & \text { if } k=4 \\ 0.3, & \text { if } k=5\end{cases}
$$


Clearly $X_{1} \leq_{l r} X_{2}$. For $\alpha=5$, we have the mass functions of $Y_{1}$ and $Y_{2}$ respectively as

$$
g_{1}(k ; 5)=\left\{\begin{array}{cl}
0, & \text { if } k=1 ; \\
\frac{3}{38}, & \text { if } k=2 ; \\
\frac{50}{209}, & \text { if } k=3 ; \\
\frac{25}{77}, & \text { if } k=4 ; \\
\frac{5}{14}, & \text { if } k=5,
\end{array}\right.
$$

and

$$
g_{2}(k ; 5)= \begin{cases}0, & \text { if } k=1 \\ \frac{1}{21}, & \text { if } k=2 ; \\ \frac{5}{42}, & \text { if } k=3 ; \\ \frac{5}{33}, & \text { if } k=4 \\ \frac{15}{22}, & \text { if } k=5\end{cases}
$$

This shows that $Y_{1} \underset{\mathbf{L}_{l r}}{ } Y_{2}$.

Counterexample 4.4 Take the random variables $X_{1}$ and $X_{2}$ having respective mass functions

$$
f_{1}(k)= \begin{cases}0, & \text { if } k=1 \\ 0.3, & \text { if } k=2 ; \\ 0.3, & \text { if } k=3 ; \\ 0.2, & \text { if } k=4 ; \\ 0.2, & \text { if } k=5,\end{cases}
$$

and

$$
f_{2}(k)= \begin{cases}0, & \text { if } k=1 \\ 0.2, & \text { if } k=2 \\ 0.3, & \text { if } k=3 \\ 0.24, & \text { if } k=4 \\ 0.26, & \text { if } k=5\end{cases}
$$

Clearly $X_{1} \leq_{l r} X_{2}$. For $\alpha=0.2$, we have the mass functions of $Y_{1}$ and $Y_{2}$ as

$$
g_{1}(k ; 0.2)= \begin{cases}0, & \text { if } k=1 ; \\ \frac{15}{22}, & \text { if } k=2 ; \\ \frac{75}{374}, & \text { if } k=3 ; \\ \frac{25}{357}, & \text { if } k=4 ; \\ \frac{1}{21}, & \text { if } k=5\end{cases}
$$


and

$$
g_{2}(k ; 0.2)= \begin{cases}0, & \text { if } k=1 \\ \frac{5}{9}, & \text { if } k=2 \\ \frac{5}{18}, & \text { if } k=3 \\ \frac{10}{99}, & \text { if } k=4 \\ \frac{13}{198}, & \text { if } k=5\end{cases}
$$

This shows that $Y_{1} \not \varliminf_{l r} Y_{2}$.

We summarize the above findings in Table 2.

Table 2: Preservation of stochastic orderings

\begin{tabular}{|l|l|l|}
\hline $\begin{array}{l}\text { Stochastic orders between } \\
\text { baseline distributions }\end{array}$ & \multicolumn{1}{|c|}{$\alpha<1$} & $\alpha>1$ \\
\hline Usual stochastic order & Preserved & Preserved \\
Hazard rate order & Not Preserved & Preserved \\
Reversed hazard rate order & Preserved & Not Preserved \\
Likelihood ratio order & Not Preserved & Not Preserved \\
\hline
\end{tabular}

\section{Conclusion}

Marshall and Olkin [19] introduced a method of adding a new parameter, called tilt parameter, to a family of distributions for obtaining more flexible new families of distributions. In the literature, some reliability properties of this family of distributions are studied with continuous baseline distributions. However, not much study is done in the literature, to the best of our knowledge, for discrete baseline distributions. This paper discusses various stochastic ageing properties, as well as different stochastic orderings of this family with discrete baseline distributions.

\section{Acknowledgements:}

The support received from IISER Kolkata to carry out this research work is gratefully acknowledged by Pradip Kundu. The financial support from NBHM, Govt. of India (vide Ref. No. 2/48(25)/2014/NBHM(R.P.)/R\&D II/1393 dt. Feb. 3, 2015) is duly acknowledged by Asok K. Nanda. 


\section{References}

[1] S. Bennett, Analysis of survival data by the proportional odds model, Statistics in Medicine 2 (1983) 273-277.

[2] C. Bracquemond, O. Gaudoin, A survey on discrete lifetime distributions, International Journal of Reliability, Quality and Safety Engineering 10(1) (2003) 69-98.

[3] C. Caroni, Testing for the Marshall-Olkin extended form of the Weibull distribution, Statistical Papers 51 (2010) 325-336.

[4] D. Collett, Modelling survival data in medical research, 2nd edition, Chapman and Hall/CRC, 2004.

[5] G.M. Cordeiro, A.J. Lemonte, E.M.M. Ortega, The Marshall-Olkin family of distributions: mathematical properties and new models, Journal of Statistical Theory and Practice 8(2) (2014) 343-366.

[6] G.M. Cordeiro, A.J. Lemonte, On the Marshall-Olkin extended Weibull distribution, Statistical Papers 54(2) (2013) 333-353.

[7] E.G. Déniz, J.M. Sarabia, A discrete distribution including the Poisson, Communications in Statistics - Theory and Methods 45(8) (2016) 2311-2322.

[8] G.E. Dinse, S.W. Lagakos, Regression analysis of tumour prevalence data, Applied Statistics 32(3) (1983) 236-248.

[9] I. Dewan, K.K. Sudheesh, Ageing concepts for discrete data- A relook, In: Proceedings of the IEEE International Conference on Quality and Reliability, Bangkok, Thailand, 2011.

[10] J.D. Esary, A.W. Marshall, F. Proschan, Shock models and wear processes, The Annals of Probability 1(4) (1973) 627-649.

[11] E. Fagiuoli, F. Pellerey, Preservation of certain classes of life distributions under Poisson shock models, Journal of Applied Probability 31 (1994) 458-465.

[12] M.E. Ghitany, E.K. Al-Hussaini, R.A. Al-Jarallah, Marshall-Olkin extended Weibull distribution and its application to censored data, Journal of Applied Statistics 32(10) (2005) 1025-1034.

[13] M.E. Ghitany, F.A. Al-Awadhi, L.A. Alkhalfan, Marshall-Olkin extended Lomax distribution and its application to censored data, Communications in Statistics - Theory and Methods 36(10) (2007) 1855-1866. 
[14] M.E. Ghitany, S. Kotz, Reliability properties of extended linear failure rate distributions, Probability in the Engineering and Informational Sciences 21 (2007) 441-450.

[15] P.L. Gupta, R.C. Gupta, R.C. Tripathi, On the monotonic properties of discrete failure rates, Journal of Statistical Planning and Inference 65 (1997) 255-268.

[16] R.C. Gupta, S. Lvin, C. Peng, Estimating turning points of the failure rate of the extended Weibull distribution, Computational Statistics and Data Analysis 54 (2010) 924-934.

[17] S.N.U.A. Kirmani, R.C. Gupta, On the proportional odds model in survival analysis, Annals of the Institute of Statistical Mathematics 53(2) (2001) 203-216.

[18] X. Li, M. Xu, Reversed hazard rate order of equilibrium distributions and a related ageing notion, Statistical Papers 49 (2008) 749-767.

[19] A.W. Marshall, I. Olkin, A new method of adding a parameter to a family of distributions with applications to the exponential and Weibull families, Biometrika 84 (1997) 641-652.

[20] A.W. Marshall, I. Olkin, Life Distributions. Springer, New York, 2007.

[21] T. Nakagawa, S. Osaki, The Discrete Weibull Distribution, IEEE Transactions on Reliability, R-24(5) (1975) 300-301.

[22] A.K. Nanda, S. Das, Some ageing properties of Marshall-Olkin extended distribution, International Journal of Mathematics and Statistics. 13(1) (2013) 93-107.

[23] A.K. Nanda, S. Das, Stochastic orders of the Marshall-Olkin extended distribution, Statistics and Probability Letters 82 (2012) 295-302.

[24] A.K. Nanda, D. Sengupta, Discrete life distributions with decreasing reversed hazard, Sankhyā 55 (2005) 164-168.

[25] A.N. Pettitt, Proportional odds models for survival data and estimates using ranks, Applied Statistics 33(2) (1984) 169-175.

[26] A.J. Rossini, A.A. Tsiatis, A Semiparametric proportional odds regression model for the analysis of current status data, Journal of the American Statistical Association 91(434) (1996) 713-721.

[27] A.A. Salvia, R.C. Bollinger, On discrete hazard functions, IEEE Transactions on Reliability 31 (5) (1982) 458-459.

[28] M. Shaked, J.G. Shanthikumar, J.B. Valdez-Torres, Discrete hazard rate functions, Computers and Operations Research 22 (4) (1995) 391-402. 